Revue internationale P.M.E.

Économie et gestion de la petite et moyenne entreprise

\title{
La propriété intellectuelle et les PME : quels enjeux et quelles pratiques?
}

\section{Olivier Crevoisier, Luc Amgwerd et Nathalie Tissot}

Volume 18, numéro 2, 2005

URI : https://id.erudit.org/iderudit/1008474ar

DOI : https://doi.org/10.7202/1008474ar

Aller au sommaire du numéro

Éditeur(s)

Presses de l’Université du Québec

ISSN

0776-5436 (imprimé)

1918-9699 (numérique)

Découvrir la revue

Citer cet article

Crevoisier, O., Amgwerd, L. \& Tissot, N. (2005). La propriété intellectuelle et les PME : quels enjeux et quelles pratiques? Revue internationale P.M.E., 18(2),

9-41. https://doi.org/10.7202/1008474ar

\section{Résumé de l'article}

La propriété intellectuelle (PI) fait l'objet d'une attention grandissante dans les stratégies des grandes entrep]rises ainsi que dans le cadre du commerce international (signature des Accords sur les aspects des droits de propriété intellectuelle qui touchent au commerce - ADPIC). Mais qu'en est-il des PME au regard de ces préoccupations ? La PI est-elle perçue comme un enjeu pour elles ? Quelles pratiques de PI ont-elles développées au regard de la nécessité d'innover et de l'utilisation de plus en plus intensive des ressources immatérielles ?

Les réponses apportées dans cette contribution sont de nature exploratoire, basée sur une enquête par entretiens menée dans la région de l'Arc jurassien. Il s'avère que la propriété intellectuelle est un instrument incomplet pour les PME dans le sens où il n'offre pas de protection absolue, loin de là. Cependant, la protection n'est pas l'unique fonction que remplissent ces outils ; leur utilisation correspond à différentes logiques qui sont mises en évidence dans la première partie. Enfin, ces logiques se combinent de différentes manières selon le type d'entreprise et leur situation.

En montrant la diversité des utilisations possibles et leurs diverses combinaisons, cet article révèle que la PI, malgré ses limites, peut être un atout pour les PME.
Ce document est protégé par la loi sur le droit d'auteur. L'utilisation des services d’Érudit (y compris la reproduction) est assujettie à sa politique d'utilisation que vous pouvez consulter en ligne.

https://apropos.erudit.org/fr/usagers/politique-dutilisation/ 


\title{
La propriété intellectuelle et les PME: quels enjeux et quelles pratiques?
}

Olivier CREVOISIER Luc AMGWERD

Nathalie TISSOT

Université de Neuchâtel

\author{
MOTS CLÉS
}

\section{PME - Propriété intellectuelle - Économies de la grandeur Innovation - Communication - Veille technologique Transfert de technologie - Concurrence}

\begin{abstract}
RÉSUMÉ
La propriété intellectuelle (PI) fait l'objet d'une attention grandissante dans les stratégies des grandes entrep]rises ainsi que dans le cadre du commerce international (signature des Accords sur les aspects des droits de propriété intellectuelle qui touchent au commerce - ADPIC). Mais qu'en est-il des PME au regard de ces préoccupations? La PI est-elle perçue comme un enjeu pour elles? Quelles pratiques de PI ont-elles développées au regard de la nécessité d'innover et de l'utilisation de plus en plus intensive des ressources immatérielles?
\end{abstract}

Les réponses apportées dans cette contribution sont de nature exploratoire, basée sur une enquête par entretiens menée dans la région de l'Arc jurassien. II s'avère que la propriété intellectuelle est un instrument incomplet pour les PME

\section{LES AUTEURS}

Olivier CRevoisier est directeur de recherches en économie régionale à l'Institut de recherches économiques et régionales (IRER). Ses recherches portent sur le développement régional et urbain, les milieux innovateurs, les circuits financiers et l'économie de la connaissance. Courriel : <olivier.crevoisier@unine.ch>.

LUC AMGWERD est assistant en droit. L'assistanat et ses recherches se concentrent sur la propriété intellectuelle, les nouvelles technologies et l'impact de ces outils sur l'environnement social et économique actuel. Courriel : <luc.amgwerd@unine.ch>.

NATHALIE TISSOT est professeure de droit de la propriété intellectuelle et de droit des nouvelles technologies de l'information et de la communication. En parallèle de son activité universitaire, elle est avocate spécialisée en droit de la propriété intellectuelle. Courriel: $<$ nathalie.tissot@span.ch>. 
dans le sens où il n'offre pas de protection absolue, loin de là. Cependant, la protection n'est pas l'unique fonction que remplissent ces outils; leur utilisation correspond à différentes logiques qui sont mises en évidence dans la première partie. Enfin, ces logiques se combinent de différentes manières selon le type d'entreprise et leur situation.

En montrant la diversité des utilisations possibles et leurs diverses combinaisons, cet article révèle que la PI, malgré ses limites, peut être un atout pour les PME.

\section{ABSTRACT}

The intellectual property (IP) is considered as a growing point of interest in the strategies of the big firms and in the context of the international trade (recent signature of the TRIPS, Trade-Related Aspects of Intellectual Property Rights). What about the SMEs, regarding those preoccupations? Are IP tools a real challenge for them? What practices did the SME develop, considering the need to innovate and the growing use of immaterial resources?

The answers, through this article, are from an explorative nature and based on a survey, carried out by interviews led in the regional of the Swiss Jura. It turns out that IP is an incomplete tool for SMEs, in the meaning that it does not offer an absolute protection. Nevertheless, the protection is not from far the unique function that fill those tools. Their use fits different logics, which are underlined in the first part of the article. Finally, those logics organize themselves in different manners, according to the type of SMEs and their situation.

By showing the diversity of potential uses and their combinations, this article suggests that IP, in spite of their inherent limits, can be an asset for SMEs.

\section{RESUMEN}

La propriedad intellecual $(P I)$ va tomando una particular relevancia tanto en la estrategia de las grandes empresas como en materia de comercio internacional (recientes negociaciones en la OMC sobre los Acuerdos sobre les Aspectos de los Derechos de Propriedad Intelectual relacionados con el Comercio, "ADPIC»). ¿Cómo se enfrentan las PyMEs a esta preocupación? ¿Constituyen los derechos de PI un desafío para ellas? ¿Cómo utilizan las PyMEs la PI en relación con sus necesidades de innovación y el uso creciente de recursos inmateriales?

Las respuestas que propone nuestra investigación son de carácter exploratorio y se apoyan sobre entrevistas realizadas en la zona suiza del Jura, el "Arc jurassien ». Ponemos de manifiesto que la Pl es una herramienta incompleta para las PyMEs ya que no permite una protección absoluta. No obstante, la protección no parece ser la única función que cumplen estos derechos. Su utilización responde à distintas lógicas que se exponen en la primera parte de nuestro estudio. Por último, estas lógicas de comportamiento se combinan de differentes formas según el tipo de PyME y su situación. 
Al mostrar la diversidad de utilizaciones y combinaciones posibles, este artículo sugiere que, a pesar de sus imperfecciones, la PI puede constituir una ventaja estratégica para las PyMEs.

\section{ZUSAMMENFASSUNG}

Der Schutz des geistigen Eigentums (IP) wird in den Strategien von grossen Unternehmen und im internationalen Handel immer stärker thematisiert (Abkommen über die rechtlichen Aspekte geistigen Eigentums im Handel: ADPIC, englisch TRIPS). Aber wie sieht es diesbezüglich bei kleinen und mittleren Unternehmungen aus ? Welche Strategien zum Schutz des geistigen Eigentums werden entwikkelt, um dem ständigen Innovationsdruck und dem sich intensivierenden Bedarf von immateriellen Ressourcen entgegenzutreten?

Die in diesem Beitrag vorgeschlagenen Antworten haben explorativen Charakter und basieren auf einer Untersuchung mit Interviews in der Region 'Arc jurassien'. Es hat sich dabei erwiesen, dass der Schutz des geistigen Eigentums ein unvollständiges Instrument für die kleinen und mittleren Unternehmungen darstellt. Dies vor allem, weil die rechtlichen Schutzstrategien noch lange keinen absoluten Schutz vor Missbrauch bieten. Jedoch ist die Schutzfunktion nicht die einzige Aufgabe, die diese Instrumente erfüllen können. Die im ersten Teil erklärte Verwendung der Werkzeuge entspricht verschiedener logischer Ansätze. Je nach Unternehmung und Situation lassen sich diese verschiedenen Ansätze unterschiedlich kombinieren und einsetzen.

Der Artikel zeigt die vielfältige Verwendung von Instrumenten zum Schutz des geistigen Eigentums auf, womit diese, trotz der erwähnten Schwächen auch zur Trumpfkarte für kleine und mittlere Unternehmen werden können.

\section{Introduction}

Comment se fait-il que les outils de propriété intellectuelle, qui ont déjà une longue histoire, soient actuellement de plus en plus au centre des préoccupations d'un éventail d'entreprises toujours plus large? La propriété intellectuelle estelle aussi l'affaire des PME? Dans le contexte économique actuel, comment ces différents droits peuvent-ils contribuer au développement de PME? Les différences entre entreprises se reflètent-elles dans leur utilisation de la PI et, dans ce cas, de quelle manière ? Cet article rapporte les résultats d'une enquête exploratoire menée conjointement par des juristes et des économistes auprès de PME et d'institutions de recherche afin de mieux cerner les enjeux et les pratiques dans ce domaine ${ }^{1}$.

1. Promotion des outils de propriété intellectuelle dans les PME de l'Arc jurassien, Projet Interreg II, Université de Neuchâtel (Prof. N. Tissot, Dr O. Crevoisier et L. Amgwerd) et Université de Franche-Comté (Prof. F. Magnin et J. Dichamp), novembre 2001.

Revue internationale P.M.E., vol. 18, n 2, 2005

(C) 2005 - Presses de l'Université du Québec

Édifice Le Delta I, 2875, boul. Laurier, bureau 450, Sainte-Foy, Québec G1V 2M2 - Tél.: (418) 657-4399 - www.puq.ca

Tiré de: Revue internationale P.M.E., vol. 18, no 2 , sous la direction de Louis Raymond • PME1802N

Tous droits de reproduction, de traduction et d'adaptation réservés 
La première section présente le cadre global de l'étude: elle énonce succinctement les grandes lignes de la propriété intellectuelle et de son utilisation croissante dans le cadre des transformations économiques et technologiques auxquelles les entreprises doivent faire face.

La deuxième décrit et justifie le cadre théorique choisi. Les économies de la grandeur (Boltanski et Thévenot, 1991) sont une approche intéressante pour la compréhension des pratiques des acteurs en situation.

La troisième regroupe ces pratiques observées dans les PME en six logiques d'utilisation relatives à un produit, une activité, une innovation ou un projet de l'entreprise. En effet, contrairement aux idées reçues, la fonction de protection, qui est généralement perçue comme la principale raison d'être de la PI, n'est de loin pas la seule utilisation qu'en font les entreprises. Le constat principal va même plus loin: la propriété intellectuelle ne devient un atout que si elle est mise en œuvre de différentes manières. Autrement dit, ces droits sont utilisés comme outils de communication, comme une technique à maîtriser (de la même manière que pourrait l'être, par exemple, la comptabilité analytique), une valeur mobilière monnayable, un outil de collaboration et, enfin, un outil de développement des connaissances.

La quatrième section cherche à répondre aux questions suivantes:

- Les logiques d'utilisation identifiées dans la section 3 sont-elles liées à des types particuliers de PME?

- Quel est le rôle (central, annexe ou subsidiaire) de la politique de PI dans le développement plus général de l'entreprise?

Cette dernière section donne lieu à l'élaboration d'une typologie non plus des logiques d'utilisation relatives à un projet, mais des profils d'entreprise en relation avec leur utilisation de la PI.

\section{La propriété intellectuelle et son rôle croissant dans l'économie contemporaine et pour les PME}

\subsection{Qu'est-ce que la propriété intellectuelle?}

La propriété intellectuelle exprime une propriété sur les créations de l'esprit. Cette notion sous-entend que l'auteur a «travaillé» ou « œuvré» pour aboutir à un résultat créatif, inventif ou particulier (voir entre autres auteurs: Merges, Menell et Lemey, 2000). Cet effort intellectuel représente la justification de la propriété et de sa protection. 
Deux principes de base ordonnent la propriété intellectuelle et permettent d'entrevoir les réels enjeux actuels de ses différents droits. En premier lieu, les portefeuilles de propriété intellectuelle permettent de «donner un visage» à des éléments qui sont immatériels (démarches artistiques, processus innovateurs, etc.). Ils finalisent donc un processus créatif ou inventif dont il est difficile, voire impossible de totalement et idéalement rendre compte. Aussi imparfaits qu'ils soient, brevets, designs, marques ou droits d'auteur permettent, par la forme juridique, de reconnaître le travail de l'auteur.

En second lieu, la propriété intellectuelle est toujours une solution de compromis. Elle tente de concilier les intérêts, de nature plutôt privée, de l'auteur et ceux du reste de la société, qui sont davantage publics. D'un côté, les titulaires des droits veulent conserver et maîtriser la propriété de leurs travaux afin de rentabiliser les investissements qui en sont à l'origine et, de l'autre côté, l'accès du public à ces créations et inventions est essentiel ${ }^{2}$. Les droits de propriété intellectuelle permettent ainsi aux auteurs de contrôler et gérer la «mise à disposition du public» de leurs œuvres, créations ou inventions.

Les différents droits font l'objet d'une présentation synthétique et systématique en annexe.

\subsection{En toile de fond: mondialisation, innovation et matérialisation}

La mondialisation des échanges économiques est à ce jour une réalité connue et répétée. Les produits sont d'une qualité sans cesse perfectionnée et perfectionnable. Ce phénomène soulève donc inévitablement de nouveaux défis pour les entreprises: il s'agit non seulement de rester à la pointe de l'innovation, mais également de maîtriser les nouvelles incertitudes liées à cette course en avant. La propriété intellectuelle s'est révélée au fil des ans un outil souvent utile, mais surtout, de plus en plus en plus indispensable pour affronter ces enjeux.

2. L'accessibilité «accordée» au public est légitimée par le souci du public de «se nourrir» des inventions et créations antérieures. Cette notion s'exprime différemment selon les droits en question, et ce, à deux niveaux. Premièrement, l'accès se définit par un faisceau de prérogatives «usuelles» relatifs à chaque droit (par exemple, la reproduction d'une œuvre protégée par le droit d'auteur, la divulgation du brevet et son contenu, etc.). Deuxièmement, des situations d'exception sont prévues, légalement, pour chacun des droits (utilisation à des fins privées en droit d'auteur, licence obligatoire en matière de brevet de l'art. 31 ADPIC...). À la différence des prérogatives «normales » évoquées ci-dessus, ces dernières ne sont admises et interprétées que de manière stricte et précise.

Revue internationale P.M.E., vol. 18, nº 2, 2005

(c) 2005 - Presses de l'Université du Québec

Édifice Le Delta I, 2875, boul. Laurier, bureau 450, Sainte-Foy, Québec G1V 2M2 • Tél.: (418) 657-4399 - www.puq.ca

Tiré de: Revue internationale P.M.E., vol. 18, $\mathrm{n}^{\circ} 2$, sous la direction de Louis Raymond • PME1802N

Tous droits de reproduction, de traduction et d'adaptation réservés 
Face à une concurrence disposant de réservoirs de main-d'œuvre à bas coûts, les pays à coûts élevés sont contraints de baser leur compétitivité sur l'innovation. Cette dernière permet quelquefois aux produits/services ${ }^{3}$ d'être meilleur marché grâce à une nouvelle technique, mais surtout d'offrir autre chose et d'échapper ainsi et pour un certain temps, à une pression sur les prix. Cependant, les innovations sont en général facilement imitables. Dès lors, la propriété intellectuelle joue un rôle important en protégeant ces «nouveautés» ou «originalités $»^{4}$. Sans cette protection, l'imitation, à coûts forcément moindres, découragerait rapidement les innovateurs et les investissements nécessaires à l'innovation devraient se faire à fonds perdus.

Parallèlement à cette montée de l'innovation et en renforcement de cette tendance, l'économie devient de plus en plus immatérielle, de plus en plus basée sur des symboles, sur la marchandisation de la culture et des expériences de vie (De Rosnay, 2000; Rifkin, 2000). La fabrication, à savoir la transformation physique de la matière, a toujours été la principale source de valeur ajoutée dans des processus industriels «classiques»; cependant, aujourd'hui, les aspects technologiques, esthétiques et liés à l'image de marque complètent, de manière importante, cette plus-value. Ainsi, le coût des opérations physiques visant à fabriquer une bouteille de vin, une montre, une machine ou un CD sont marginaux par rapport aux coûts de constitution des savoir-faire, de recherche et de développement, de maturation artistique, d'investissement dans l'image de marque... Très souvent, un maillot de bain n'est plus un simple bout de tissu imperméable et coupé correctement pour remplir sa fonction première; il intègre des recherches sur le textile (séchage rapide, «adhérence» à l'eau, solidité, etc.), des réflexions esthétiques et culturelles relevant de la mode et sur l'image de marque. Cette valeur ajoutée immatérielle est produite par des services liés aux connaissances technologiques, scientifiques, artistiques ou de nature plus sociologique ou publicitaire pour ce qui est de l'image donnée à un produit ou service. La propriété intellectuelle permet donc de faire de ces connaissances ou de ce savoir-faire un «outil», un bien marchand à part entière.

Ces grandes tendances s'imposent naturellement aux PME. Cependant, les outils de PI semblent a priori bien mal adaptés à leur réalité. En effet, la formalisation juridique est coûteuse en plus de requérir des compétences spécifiques.

3. Nous proposons d'abandonner les traditionnelles distinctions entre les notions de biens et de services, entre produits et prestations, pour regrouper tout type d'offre économique sous le terme générique de produit. Outre l'allègement de texte que ce regroupement permet, il nous semble être en phase avec le langage que bon nombre d'entrepreneurs ou prestataires de services utilisent.

4. Les droits de propriété intellectuelle accordent un monopole temporaire d'exploitation sur de nouveaux produits ou de nouvelles technologies.

Revue internationale P.M.E., vol. 18, nº 2, 2005

(C) 2005 - Presses de l'Université du Québec

Édifice Le Delta I, 2875, boul. Laurier, bureau 450, Sainte-Foy, Québec G1V 2M2 • Tél.: (418) 657-4399 - www.puq.ca

Tiré de: Revue internationale P.M.E., vol. 18, $\mathrm{n}^{\circ} 2$, sous la direction de Louis Raymond - PME1802N

Tous droits de reproduction, de traduction et d'adaptation réservés 
Les PME ont des difficultés à mettre en œuvre une politique de propriété intellectuelle complète (large éventail de dépôts et défense des droits) et sont très hétérogènes (Working Paper, 2001), avec une palette de comportements divers en termes d'innovation, de création, de gestion ou de marketing. Il est dès lors primordial pour ces entités de réussir à connaître au sein même de l'entreprise, dans un premier temps, les enjeux réels de ces outils et d'être à même par la suite de mobiliser les compétences nécessaires et efficaces dans leur environnement.

\section{Présentation de l'enquête et cadre théorique}

Les PME ont recours de plus en plus aux différents outils de PI. Cependant, les raisons qui les y amènent ne sont guère connues. Alors même que les outils de PI sont incomplets, imparfaits (voir entre autres Amable, Barré et Boyer, 1997; Héraud 1995; Davis, 2001; Le Bas, 1999; Deffains, 1997) et généralement coûteux à défendre, pourquoi une entreprise choisit-elle de protéger tel ou tel dispositif technique, tel ou tel modèle? Comment ce choix s'inscrit-il dans la problématique plus générale de l'entreprise?

Nous sommes partis de l'idée qu'il n'existe pas de politique standard de la PI, pas plus qu'il n'existe de PME standard. Cela dit, notre problème consistait à rapporter la manière dont les entreprises expliquent et justifient leurs choix et à dégager parmi ces propos et ces expériences un certain nombre de logiques cohérentes. Dès lors, quel cadre théorique et quelle méthode choisir?

Étant donné que nous avons choisi de travailler par entretiens et de demander aux acteurs eux-mêmes comment ils justifiaient leurs pratiques, l'approche des économies de la grandeur (Boltanski et Thévenot, 1991) était appropriée. Les justifications qui nous étaient livrées peuvent, selon ces auteurs, être regroupées en un nombre restreint de catégories. Dans leur ouvrage cité et pour la France de 1990, ils relevaient six «mondes»; chacun d'eux est régi par un principe supérieur et révèle ainsi une «certaine logique» des acteurs dans leurs rapports (avec d'autres personnes, avec le passé, avec l'incertitude...) et donc dans des situations concrètes (tableau 1).

À partir de cette approche, il n'est pas possible de dégager une seule «bonne recette» quant à la manière de recourir à la PI. En revanche, nous avons pu clairement identifier un certain nombre de logiques d'utilisation de la PI par les PME. Il a été intéressant d'observer le «résultat» de la tension entre le formalisme des règles de droit et les incertitudes de l'innovation. Trouver de quoi est faite cette «boîte noire» et tenter de donner une lecture compréhensible des pratiques était l'objectif principal de l'étude.

Revue internationale P.M.E., vol. 18, nº 2, 2005

(c) 2005 - Presses de l'Université du Québec

Édifice Le Delta I, 2875, boul. Laurier, bureau 450, Sainte-Foy, Québec G1V 2M2 • Tél.: (418) 657-4399 - www.puq.ca

Tiré de: Revue internationale P.M.E., vol. 18, $\mathrm{n}^{\circ} 2$, sous la direction de Louis Raymond • PME1802N

Tous droits de reproduction, de traduction et d'adaptation réservés 
Le dépouillement des entretiens a été réalisé dans un premier temps à partir de ces six «mondes», qui se sont d'ailleurs révélés très pertinents. Nous n'avons par conséquent pas éprouvé le besoin d'ajouter de nouveaux «mondes» ou d'en retrancher certains qui ne seraient pas apparus comme pertinents. Cependant, dans un deuxième temps, il a été nécessaire de décrire ces mondes pour parler des pratiques de la PI. Pour prendre un exemple, Boltanski et Thévenot (1991) parlent du «monde de l'opinion» pour regrouper les pratiques qui se justifient pour gagner en notoriété, par la publicité, la communication, etc. Dans le domaine de la PI, la dimension de la communication est présente de diverses manières : publicité des brevets, notoriété des marques, veille technologique, etc. Nous avons parlé de la PI comme d'un «outil de communication». Il a ainsi été possible de réunir l'essentiel de nos données sous six logiques d'utilisation qui se rapprochent des mondes de Boltanski et Thévenot tout en ayant un contenu concret plus caractéristique de la problématique observée (tableau 1).

L'approche de Boltanski et Thévenot permet, en outre, à travers les notions de «grand» ou de «petit» dans un monde ou dans un autre de rendre compte de l'évaluation souvent ambivalente que l'on peut faire de la PI. Les ambiguïtés ont été ressenties à de nombreuses reprises lors des entretiens, les interlocuteurs relevant tantôt des expériences positives, tantôt peu concluantes ou négatives. Par exemple, la PI peut être perçue comme facilitant la coopération en précisant les droits et les devoirs de chacun, mais, à l'inverse, elle peut «plomber juridiquement» une relation entre des acteurs économiques et induire la méfiance entre eux; ou encore, le brevet protège l'invention, mais s'accompagne d'une diffusion des caractéristiques techniques et, par conséquent, facilite son contournement ou son utilisation par les concurrents...

Concrètement, l'étude a été menée conjointement dans l'Arc jurassien suisse et français et a duré deux ans. La partie suisse de l'étude, dont le présent article rend plus particulièrement compte, a touché $24 \mathrm{PME}$ et institutions de recherche et formation. Le choix des entreprises s'est fait selon quatre critères:

- entreprises qui sont, ou ont été, confrontées à des questions de propriété intellectuelle;

- entreprises employant moins de 250 personnes ou institutions de recherche;

- localisées dans la région de l'Arc jurassien suisse;

- actives dans les branches horlogère, mécanique, microtechnique, agroalimentaire et dans l'industrie du bois. 


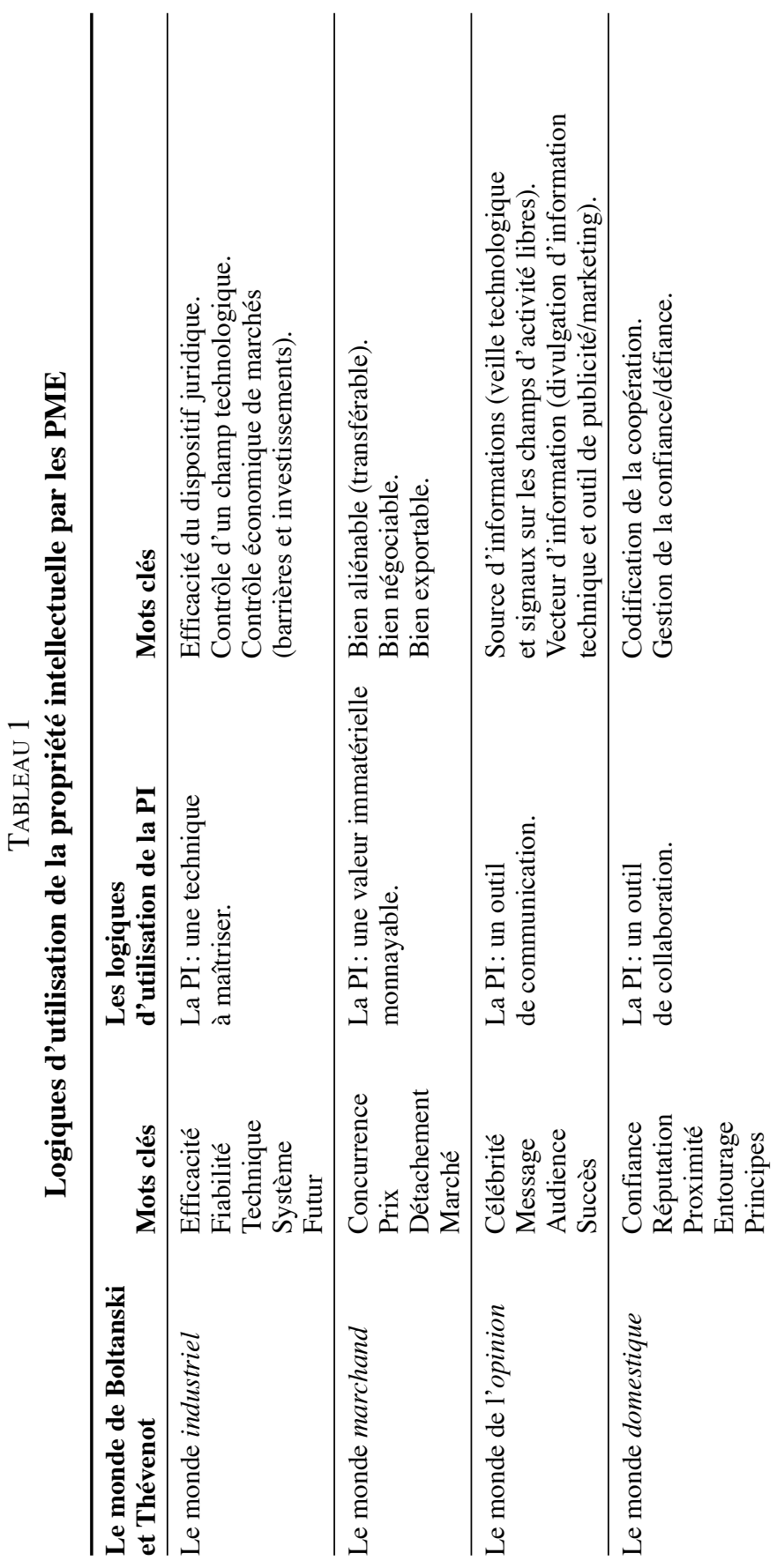

Revue internationale P.M.E., vol. 18, nº 2, 2005 


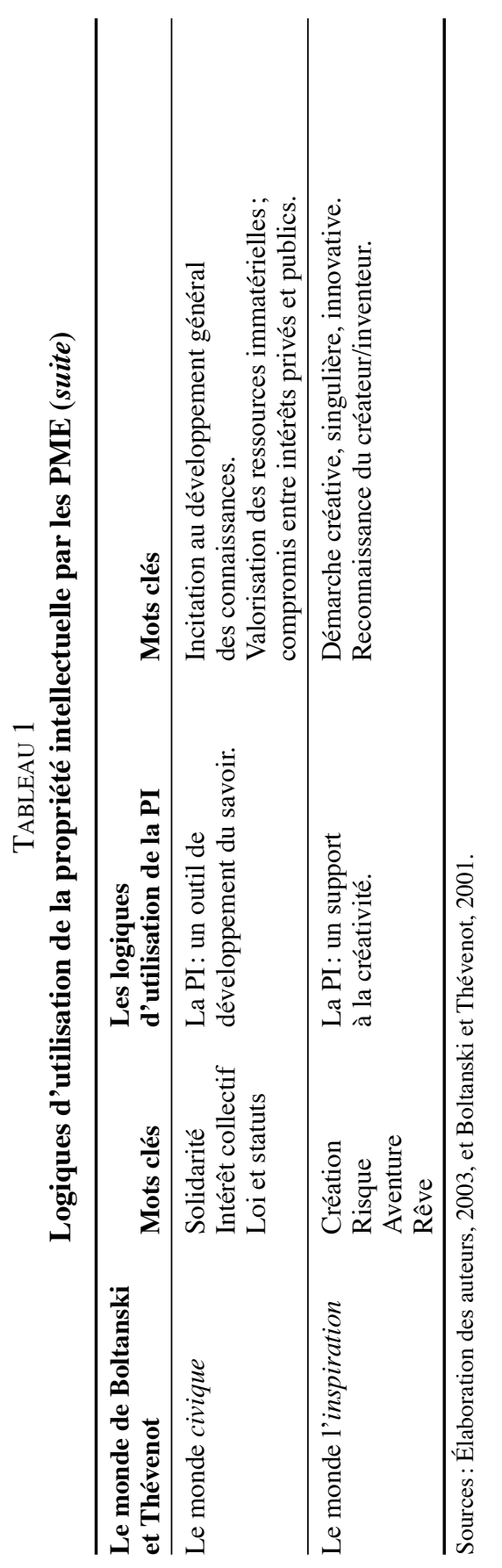

Revue internationale P.M.E., vol. 18, $\mathrm{n}^{\circ}$ 2, 2005 
Les entretiens se sont déroulés sous forme d'interviews semi-directs, en parcourant les questions d'un guide élaboré au préalable. Nous avons généralement disposé de plus d'une heure pour aborder la problématique.

Les buts généraux de l'étude relevaient de trois ordres. Dans un premier temps, il importait de faire un état des lieux des pratiques observées de la propriété intellectuelle chez les principaux acteurs de la région dans les différents domaines industriels abordés. Il n'a nullement été question d'aboutir à des résultats statistiques et ayant une quelconque valeur quantitative. Dans un deuxième temps, il s'avérait nécessaire de tirer une réflexion pertinente et, somme toute, aussi proche que possible des réalités vécues par les entreprises et les institutions de recherche. Enfin, nous désirions proposer, aux PME, des clés ou des pistes pour une valorisation judicieuse et efficace de leurs créations intellectuelles

\section{La propriété intellectuelle en situation: six logiques d'utilisation}

\subsection{La $\mathrm{PI}$, une technique à maîtriser pour être efficace}

Le brevet, la marque et les designs fixent des monopoles d'utilisation qui permettent à l'entreprise de faire des investissements sans craindre que des imitateurs ruinent ce dispositif. Ils définissent un certain nombre d'éléments pour l'action future de l'entreprise. Dans ce sens, la loi garantit à partir d'une technologie décrite un laps de temps (fixe ou renouvelable) et des espaces (rayon de validité des titres) qui définissent un champ d'action tourné vers le futur pour l'entreprise ${ }^{6}$. Elle établit des barrières, qualifiées quelquefois de «boucliers». À partir de cela, l'entreprise peut mettre sur pied des investissements de manière efficace.

Cette efficacité des droits de PI a trois aspects : juridique, technique et économique. L'efficacité juridique permet la mise en œuvre de techniques de droit (rédaction précise et détaillée des titres, conformité aux normes techniques et légales, maîtrise des délais etc.) et la défense de celles-ci. L'efficacité technique met en avant la dimension de «certification officielle» de l'existence et de la nouveauté de la création intellectuelle. Ce sont les procédures d'enregistrement ou de dépôt des droits qui confèrent à ces inventions une certaine garantie et fiabilité

6. Le brevet «offre» un outil didactique, qui peut s'appliquer par analogie aux autres droits, afin de «visualiser» les dimensions de le protection qui est garantie. Il s'agit des «3T»: technologie, temps et territoire.

Revue internationale P.M.E., vol. 18, nº 2, 2005

(c) 2005 - Presses de l'Université du Québec

Édifice Le Delta I, 2875, boul. Laurier, bureau 450, Sainte-Foy, Québec G1V 2M2 • Tél.: (418) 657-4399 - www.puq.ca

Tiré de: Revue internationale P.M.E., vol. 18, n 2, sous la direction de Louis Raymond • PME1802N

Tous droits de reproduction, de traduction et d'adaptation réservés 
technique. Enfin, l'efficacité économique résultant du droit exclusif d'exploitation pour une durée limitée, sur un territoire donné et concernant une technique précise permet à l'entreprise de prévoir et de mettre sur pied des investissements et des démarches de coordination industrielle.

Cette efficacité doit être relativisée pour les PME, car elles n'ont pas toujours les moyens financiers ni les compétences pour mettre en œuvre une politique de PI, visant une efficacité sur tous les plans. Par exemple, même si les titres de PI sont souvent bien conçus du point de vue juridique, ils peuvent être contestés, contournés et les procédures nécessaires à leur mise en œuvre peuvent être compliquées et coûteuses... Ces droits de PI sont alors perçus davantage comme des mécanismes dissuasifs, sorte de «revolver à blanc» envers d'éventuels imitateurs. Au regard de la théorie des marchés contestables (Baumol, Panzar et Willig, 1982), ils représentent des «barrières» à un marché, rendant l'accès à celui-ci contraignant et conditionné à différents investissements (coûts, temps, etc.).

Les «grandes» PME, ainsi que des entreprises au domaine d'activité très délimité (c'est le cas des spin-off, par exemple), se révèlent capables de faire des titres de PI des instruments efficaces dans ce sens. Dès lors, on pourrait penser que, sauf exception, la PI est un outil peu adapté aux PME, en général. C'est le cas si l'on en reste à l'idée reçue que la PI n'a qu'un rôle défensif. Pour le reste, notre enquête a montré que cette incomplétude de la fonction protectrice n'empêche pas les PME d'utiliser la PI de manière cohérente et efficace, mais en lui conférant d'autres fonctions qui sont présentées ci-dessous.

\section{La nécessité de brevets dans le développement médical}

Nous avons rencontré des responsables de PME spécialisées dans le développement d'équipement médical. Leur politique de brevet n'est pas étrangère au succès de l'entreprise. «Le brevet est vital dans notre domaine d'activité.» Non seulement le client veut une assurance fiable, mais le produit, intégrant l'invention met aussi du temps à être rentable. Pour ces deux raisons essentielles, nos interlocuteurs reconnaissent que leur «famille de brevets est un pivot pour leur PME». Au fil des années d'activités, les brevets sont également devenus des éléments motivants pour eux et leurs collaborateurs: motivation à «ne pas s'endormir» sur la fierté et les rentes des premiers brevets et à l'observation des nouvelles pratiques scientifiques et tendances du marché.

\subsection{La PI, une valeur immatérielle monnayable}

La PI est-elle le reflet d'une marchandisation des connaissances? En quelque sorte. Cependant, soyons attentifs à deux éléments. D'une part, la description du savoir dans le titre de PI rend les connaissances «objectives»; d'autre part, la PI

Revue internationale P.M.E., vol. 18, nº 2, 2005

(C) 2005 - Presses de l'Université du Québec

Édifice Le Delta I, 2875, boul. Laurier, bureau 450, Sainte-Foy, Québec G1V 2M2 • Tél.: (418) 657-4399 - www.puq.ca

Tiré de: Revue internationale P.M.E., vol. 18, $\mathrm{n}^{\circ} 2$, sous la direction de Louis Raymond PME1802N

Tous droits de reproduction, de traduction et d'adaptation réservés 
confère des droits de propriété sur ces «objets» immatériels et, par conséquent, rend possible leur échange sur des marchés. L'identification du contenu d'un titre de PI favorise l'évaluation de sa valeur économique et les titres de PI acquièrent donc une valeur marchande négociable et susceptible de circuler.

Les titres de PI qui «objectivisent» ainsi des connaissances ou des biens immatériels peuvent être insérés plus aisément dans un bilan, dans un plan d'affaires: ils deviennent des «biens» marchands, monnayables et négociables. Ils s'adaptent dès lors aux règles du marché et peuvent circuler dans un espace dont les normes sont de plus en plus globalisées. C'est indubitablement une tendance actuelle pour nombre de PME (Working Paper, 2001).

Autre fonction marchande intéressante, les titres de PI sont pour les PME des «passeports» pour entrer en négociation avec des concurrents ou partenaires industriels. Si certaines PME font de la PI un usage avant tout défensif, de protection, d'autres y recourent intensément pour faire le commerce de leurs idées et de leurs compétences. Il s'agit certainement d'un type nouveau d'entreprises qui a pu se développer à partir de la diffusion et de l'usage de la PI. Ce sont des entreprises généralement jeunes, actives dans plusieurs domaines. Elles vendent des «idées» de toutes sortes et leur principal capital est leur «matière grise». La PI «matérialise» leurs travaux, forge leur identité et est au cœur des procédures organisationnelles de l'entreprise.

Dans les schémas plus traditionnels de valorisation des résultats des centres de recherche, la PI permet également d'entrer en négociation avec des investisseurs - qui sont souvent de grands groupes. Dans ces cas, le brevet apparaît comme un instrument inévitable dans la recherche de financements lors du passage d'un projet scientifique à un projet industriel.

La marque traduit également une valeur qui peut être monnayable; elle est une sorte de "goodwill» de l'entreprise et de ses produits et services. Dans les secteurs d'activité en rapport direct avec les consommateurs, la renommée ou l'image de marque représente une grande partie de la valeur de la société. Les exemples des industries horlogère et alimentaire en témoignent largement au regard des moyens mis en œuvre pour lutter contre la contrefaçon et des dédommagements demandés: quel est le préjudice (le dommage en termes matériels et de l'image) porté à une marque, si un million de contrefaçons de bonne qualité se vendent sur le marché?

Ainsi, les droits de PI deviennent, dans nombre de situations, un bien économique en tant que tel, «traditionnel» et identifiable: il se négocie, s'échange, s'incorpore dans un plan d'affaires et participe à fonder la stratégie commerciale de l'entreprise. Cependant, à l'extrême, la PI est un mécanisme qui «s'oppose » au marché: ces droits permettent d'asseoir des monopoles, de contrôler des marchés

Revue internationale P.M.E., vol. 18, nº 2, 2005

(c) 2005 - Presses de l'Université du Québec

Édifice Le Delta I, 2875, boul. Laurier, bureau 450, Sainte-Foy, Québec G1V 2M2 • Tél.: (418) 657-4399 - www.puq.ca

Tiré de: Revue internationale P.M.E., vol. 18, $\mathrm{n}^{\circ} 2$, sous la direction de Louis Raymond • PME1802N

Tous droits de reproduction, de traduction et d'adaptation réservés 
et d'entraver l'innovation. Les grandes entreprises en particulier font souvent un tel usage de ces droits. À titre d'anecdote, rappelons-nous qu'historiquement (Brenner, 1998; Héraud, 1995 ; Primo Barga, Fink et Paz Sepuldeva, 2000) le brevet a été mis en œuvre afin d'assurer à la Vénétie le monopole du travail du verre, attirant du même coup des artisans qualifiés et inventifs dans ce domaine...

\subsection{La PI, un outil de communication}

Encore récemment, les droits de PI étaient présentés comme de simples outils de protection et d'exclusion. Autrefois perçue comme une tare (Foray, 1995; Granstrand, 1999; Working Paper, 2001), la communication de données techniques mérite aujourd'hui une attention particulière au sein des PME. Ainsi, les portefeuilles de PI sont tantôt utilisés comme des vecteurs, tantôt comme des sources d'informations. Ils permettent à la fois de diffuser un message relatif aux produits de la PME ou à l'entreprise elle-même et d'accéder, de manière fiable et aisée, à l'information concernant les concurrents. En plus d'être des «signes de ralliement ou d'identification» pour les consommateurs, clients, concurrents, partenaires ou autres intéressés, ils s'inscrivent de plus en plus dans une logique de communication «à double sens».

Dans un premier temps donc, les portefeuilles de PI sont utilisés par les PME comme sources d'informations. Cela se fait naturellement par la consultation, plus souvent épisodique que régulière, des bases de données technologiques et le dépouillement systématique des brevets de la concurrence. Dans quelques rares cas, certains employés sont chargés de la veille, alors que quelques PME de pointe sous-traitent ce service, généralement à un courtier en informations. Cette veille technologique permet aux PME de connaître l'état d'un «champ» d'activités. Où se situent les limites dans l'exploitation d'une idée, d'une technique, d'une image? Convient-il de s'en inspirer, de contourner les droits existants ou d'abandonner le domaine? Quelle est la liberté d'utilisation? Ces titres de PI fonctionnent comme des «repères dans un radar» permettant d'identifier les libertés d'exploitation éventuelles.

Un autre apport de la diffusion des savoirs contenus dans ces titres est de servir de «marche-pied » pour de nouvelles idées : les chercheurs et artistes s'appuient sur ces connaissances existantes et codifiées pour imaginer de nouveaux projets, lancer des développements complémentaires ou combiner des techniques entre elles. C'est un élément central de la veille technologique. C'est un moyen aisé et peu coûteux d'accéder à des sources de connaissances fraîches et fiables.

Dans un deuxième temps, la PI, tout particulièrement le brevet et la marque, est également un vecteur d'informations. Ces titres véhiculent une image spécifique, permettant aux consommateurs de l'associer à une prestation ou une 
entreprise. Le brevet reflète une image de progrès ou de créativité particulière que la PME veut promouvoir et faire connaître. La marque, pour sa part, assure la cohérence entre le produit, son image auprès des consommateurs, son design, voire ses performances. Elle participe à créer ou garantir l'identité de l'entreprise ou de ses produits ou services, en aidant à consolider un monopole sur un nom, une image (Allegrezza et Guarda-Rauchs, 1999) ou un autre signe distinctif. Il est évident que les efforts entrepris dans une perspective de type publicitaire sont d'autant plus importants que la PME est proche des consommateurs. Ainsi, pour les PME horlogères, l'investissement sur les marques et les modèles représente l'essentiel du capital de l'entreprise ! Dans un monde où la fonctionnalité première d'un bien ou d'un service n'est plus suffisante pour séduire les consommateurs, l'adjonction de composants culturels (image, histoire, etc.) est indispensable. Cela est possible grâce à la PI.

Finalement, les titres de PI sont également une façon de faire savoir aux concurrents que l'entreprise occupe déjà le terrain. Il s'agit non seulement de protéger ses créations ou inventions, mais il faut pour une entreprise afficher sa propriété afin de décourager les éventuels imitateurs. Diffuser son brevet, publier ses créations, s'exposer dans les foires, exiger des excuses publiques du contrefacteur ou usurpateur, publier ostensiblement le jugement en cas de procès, obtenir des mesures préventives spectaculaires (saisie d'une collection à des moments opportuns) sont des manières d'exister dans une logique de communication. Ces solutions sont autant de moyens de s'imposer face aux concurrents et de faire parler de sa société.

À l'inverse, la PI fait quelquefois l'objet de «non-communication». Le secret est, dans certaines circonstances, considéré comme une stratégie plus rentable que le brevet. Par exemple, lorsque, à partir de l'analyse du produit, il est difficile de remonter au procédé de fabrication, certaines entreprises préfèrent maintenir le procédé secret. En plus d'être économique, cela empêche l'imitation et le contournement du procédé par les concurrents.

\subsection{La PI, un outil de collaboration}

Dans tout processus d'innovation, de création ou de transfert technologique, la confiance est un élément essentiel. Or, elle ne se décrète pas. La PI est-elle un moyen de gagner ou d'entretenir la confiance entre partenaires? Ou, au contraire, la PI est-elle inhibante, «plombant» les relations partenariales dans le juridisme?

L'importance de la confiance marque en particulier les relations qu'entretiennent les PME et leurs partenaires spécialistes de la PI. Ces partenaires, nombreux et diversement spécialisés (avocats spécialistes en PI, ingénieurs-conseils,

Revue internationale P.M.E., vol. 18, nº 2, 2005

(C) 2005 - Presses de l'Université du Québec

Édifice Le Delta I, 2875, boul. Laurier, bureau 450, Sainte-Foy, Québec G1V 2M2 - Tél.: (418) 657-4399 - www.puq.ca

Tiré de: Revue internationale P.M.E., vol. 18, n² 2, sous la direction de Louis Raymond • PME1802N

Tous droits de reproduction, de traduction et d'adaptation réservés 
centres de recherche, partenaires industriels, etc.), sont amenés à travailler étroitement avec la PME lors de processus d'innovation. Comme de nombreuses modifications, itérations et mises au point sont nécessaires, ces différentes parties coproduisent la PI. Coopérer est une manière de gérer les incertitudes engendrées par l'innovation. L'incomplétude des brevets est compensée, en quelque sorte, par des mises en relation des acteurs (Bessy et Brousseau, 1997). Dans un tel cas, la PI est vécue comme le moteur ou l'aboutissement d'une collaboration.

La relation entre l'entreprise et son spécialiste en PI est souvent déterminante dans le succès de sa politique en la matière. Pour la PME, qui ne dispose pas, dans la grande majorité des cas, de spécialiste à l'interne dans ce domaine, la confiance dans un conseiller en PI est le seul moyen de gérer l'incertitude qui résulte de son caractère incomplet et imparfait. La continuité dans la relation et la proximité géographique sont des éléments appréciés et bénéfiques dans ce type de collaboration; elles épargnent à l'industriel des explications trop longues sur les produits/services ou le positionnement de l'entreprise sur le marché et évitent une dispersion de données techniques et commerciales propres à la PME.

La PI joue un rôle variable entre PME et instituts de recherche, ainsi qu'entre PME. Dans l'Arc jurassien, la proximité et les relations informelles sont des éléments essentiels lors de collaborations; cela rassure et facilite les contacts. Cependant, force est de constater que les PME voient fréquemment dans la PI un outil de collaboration encourageant, pourvu que les modalités actuelles et futures de la collaboration soient stipulées d'emblée et de manière à la fois précise et minimale.

Une collaboration avec les PME - morceaux choisis des Hautes Écoles spécialisées (HES)

Pour toutes les HES rencontrées, le brevet n'est pas central, mais il «fait partie de la panoplie des éléments utiles» dans la valorisation des résultats de recherche. En fait, il devient «un maillon important dans l'activité de l'institut». Bien plus qu'une valeur en soi, le brevet est souvent une «carte de visite» ou une manière de prolonger les travaux de recherche d'une équipe en une expérience industrielle. Tous nos interlocuteurs nous ont confirmé avoir assisté, ces dernières années et au sein de leur école, à la création de jeunes entreprises quand ils ne les ont pas euxmêmes lancées. Ces récents succès permettent à certains d'entre eux d'élargir et d'intensifier les activités de l'institut vers une interface entre la recherche et l'économie, devenant ainsi la «cheville ouvrière essentielle entre ces deux mondes». Nos interlocuteurs reconnaissent, d'une même voix, que «chacun des partenaires a son rôle à jouer». Ils insistent néanmoins davantage sur le changement de mentalité des industriels à leur égard. La PI aidant, les HES sont plus considérées et

Revue internationale P.M.E., vol. 18, nº 2, 2005

(C) 2005 - Presses de l'Université du Québec

Édifice Le Delta I, 2875, boul. Laurier, bureau 450, Sainte-Foy, Québec G1V 2M2 • Tél.: (418) 657-4399 - www.puq.ca

Tiré de: Revue internationale P.M.E., vol. 18, $\mathrm{n}^{\circ} 2$, sous la direction de Louis Raymond PME1802N

Tous droits de reproduction, de traduction et d'adaptation réservés 
prennent désormais part à des négociations importantes. «Les milieux industriels acceptent d'investir et de valoriser de plus en plus, ce qui laisse présager une collaboration plus intense et meilleure.»

À l'inverse, la PI peut apparaître comme un élément qui bloque ou rompt une relation de confiance dans certains cas. Chercher à formaliser juridiquement une collaboration peut soulever deux problèmes. Premièrement, imposer un dispositif de PI peut être perçu comme l'expression de soupçons quant à la loyauté du partenaire ou simplement comme un signe de méfiance. Peu importe que ce soit à tort ou à raison. Par leur côté codificateur, les droits de PI, et tout particulièrement le brevet, sont perçus comme laissant peu ou pas de place à la confiance dans des processus de transferts des connaissances (contrats de licence ou cession de brevet).

\subsection{La PI, un outil de développement du savoir}

La PI permet le développement et la diffusion du savoir et de connaissances nouvelles, et contribue ainsi à favoriser le bien de la société et à faire évoluer la science. Cependant, comment les droits de PI jouent-ils ce rôle?

Les outils de PI sont certainement des incitations à la production de connaissances : la nature exclusive de la protection accordée par ces droits permet, théoriquement, au titulaire de rentabiliser ses investissements pour de telles productions. De l'autre côté, lorsque le monopole est échu, il est possible pour tout tiers de profiter de ces inventions ou créations. Dans ce sens, le brevet est un compromis entre la jouissance individuelle du déposant et la mise à disposition du public des connaissances techniques essentielles (Amable, Barré et Boyer, 1997; Davis, 2001 ; Deffains, 1997 ; Foray, 1995 ; Héraud, 1995 ; Le Bas, 1999). La divulgation des informations contenues dans le brevet est obligatoire; elle permet donc aux tiers de les comprendre puis, éventuellement, de les utiliser et de commencer ou continuer leurs recherches sur cette base, dans leurs intérêts professionnels et dans celui de la science. Dans le même ordre d'idées, les contrats de licence permettent d'adoucir le monopole des titulaires au profit de tiers intéressés à partager l'utilisation de leurs droits exclusifs contre rémunération. Cette solution est intéressante mais se révèle souvent difficile à négocier et à exécuter dans la pratique.

Aujourd'hui, les centres de recherche sont fortement incités à valoriser leurs résultats. Les brevets en particulier offrent un cadre aux transferts du savoir. Ils ne sont pas le seul et unique canal de valorisation des résultats de recherche, mais ils sont devenus une alternative de plus en plus utilisée. Dans certaines situations, un conflit peut apparaître entre la logique marchande et la mission des instituts de

Revue internationale P.M.E., vol. 18, no 2, 2005

(C) 2005 - Presses de l'Université du Québec

Édifice Le Delta I, 2875, boul. Laurier, bureau 450, Sainte-Foy, Québec G1V 2M2 - Tél.: (418) 657-4399 - www.puq.ca

Tiré de: Revue internationale P.M.E., vol. 18, n² 2, sous la direction de Louis Raymond • PME1802N

Tous droits de reproduction, de traduction et d'adaptation réservés 
recherche de développement des connaissances pour l'ensemble de la société. Là encore, le brevet et les contrats joints sont des outils qui peuvent techniquement aboutir à une solution équitable pour l'ensemble des parties.

\subsection{La PI, un support à la créativité}

L'encouragement au développement de nouvelles connaissances et à la production artistique est inhérent à la philosophie de base de la PI. Les périodes de recherche et de création apparaissent généralement comme des moments flous et désordonnés. Dans ce processus, les droits de PI sont une «balise» pour l'ensemble des acteurs investis : un brevet ou un design représente une étape définie, un but, voire l'achèvement d'un projet.

Deux éléments semblent intéressants à relever au regard de cette logique.

Premièrement, une politique de PI est le reflet d'une démarche nouvelle ou innovatrice. Certaines des PME rencontrées créent ou cultivent cet état d'esprit créatif, sans cesse renouvelé; cependant, chacune a sa «méthode». La mise en relation et l'échange entre caractères et formations différents ont souvent été présentés comme stimulants pour une entreprise ou un institut. Dans ce cadre, la PI traduit «tant bien que mal» cet esprit: pour certains, c'est une étape nécessaire alors que d'autres, à l'opposé, estiment que le processus d'innovation se déroule très bien sans ces outils.

Deuxièmement, il est une constante que l'on ne peut taire, dans ces comportements créatifs ou innovateurs: la forte dimension personnelle des acteurs. Le rôle du chercheur, du professeur ou de l'entrepreneur qui mène le projet est déterminant: il décide, investit son temps, cherche des contacts, négocie, etc. Ainsi, les brevets sont parfois perçus comme des «bébés technologiques»! Cet attachement et l'implication personnelle qui y est liée sont non seulement caractéristiques des projets scientifiques ou artistiques, mais jouent aussi un rôle déterminant dans le succès d'une politique de PI.

\subsection{Ne pas oublier les rapports de force!}

L'approche par les économies de la grandeur permet de faire ressortir les cohérences argumentaires mobilisées par nos interlocuteurs. Cependant, cette approche ne rend pas compte des rapports de force économiques (Livian et Herreros, 1994). Or, cela est souvent apparu lors des entretiens. Il serait illusoire de croire que la PI introduirait une quelconque justice égalitaire dans les relations économiques! Ainsi, l'efficacité de la PI est limitée par les ressources dont dispose l'entreprise pour la défense de ses droits. La créativité et l'innovation peuvent être bridées par l'obtention de titres de PI par une grande entreprise visant à contrôler une

Revue internationale P.M.E., vol. 18, nº 2, 2005

(C) 2005 - Presses de l'Université du Québec

Édifice Le Delta I, 2875, boul. Laurier, bureau 450, Sainte-Foy, Québec G1V 2M2 • Tél.: (418) 657-4399 - www.puq.ca

Tiré de: Revue internationale P.M.E., vol. 18, $\mathrm{n}^{\circ} 2$, sous la direction de Louis Raymond PME1802N

Tous droits de reproduction, de traduction et d'adaptation réservés 
technique ou un marché. La confiance entre partenaires, nécessaire à tout projet d'innovation, peut être rompue entre une PME et un partenaire plus important (grande entreprise ou centre de recherche) lorsque ce dernier impose ses conditions à la PME et que cette dernière n'est pas en mesure de se défendre avec des moyens comparables.

\section{Logiques d'utilisation et types de PME}

Les logiques d'utilisation exposées ci-dessus sont centrées sur la PI et se rapportent avant tout à un projet, un produit ou une activité bien particulière de l'entreprise. Peut-on cependant dégager des logiques d'utilisation dominantes suivant le type d'entreprise? Au regard de différents critères caractérisant plus globalement le positionnement de l'entreprise le long de la chaîne de valeur, ses processus d'innovation ou encore sa relation aux marchés, il apparaît que les logiques d'utilisation de la PI se combinent de manière spécifique. Le tableau 2 croise les six types d'entreprises rencontrées avec les logiques d'utilisation de la PI, présentées ci-dessus.

\subsection{Haute technologie}

Le groupe des entreprises de haute technologie est constitué de «spin-offs» des centres de recherches publics ou privés, «spin-offs» qui doivent leur existence à un chercheur devenu entrepreneur. L'avantage compétitif de l'entreprise est précisément cette relation privilégiée avec la recherche. Lors du lancement de l'entreprise, il y a une très forte identification entre trois éléments: les connaissances issues de la recherche menée par la personne qui devient entrepreneur, la nouvelle entreprise et cette personne. Dans cette relation, les brevets jouent un rôle essentiel, car ils donnent une reconnaissance juridique à cette identité et aident ainsi le nouvel entrepreneur à se lancer.

L'avantage compétitif de l'entreprise ne réside pas seulement dans sa relation avec le marché. Ce dernier est étroit, très spécialisé, constitué généralement de grandes entreprises industrielles intéressées à incorporer des composants ou des processus de haute technologie à leurs produits. La recherche est le moteur de l'entreprise et a tendance à le rester, permettant à l'entreprise de perfectionner et d'élargir ses débouchés à l'avenir.

La PI est fondamentale pour ce type d'entreprise. Avant toute autre logique, elle est une technique à maîtriser. Le brevet protège l'invention initiale, permet des investissements, balise la technologie, ouvrant ainsi la porte très souvent à des brevets complémentaires (famille de brevets). Les titres de PI débouchent

Revue internationale P.M.E., vol. 18, n 2, 2005

(c) 2005 - Presses de l'Université du Québec

Édifice Le Delta I, 2875, boul. Laurier, bureau 450, Sainte-Foy, Québec G1V 2M2 • Tél.: (418) 657-4399 - www.puq.ca

Tiré de: Revue internationale P.M.E., vol. 18, no 2, sous la direction de Louis Raymond - PME1802N

Tous droits de reproduction, de traduction et d'adaptation réservés 
ainsi sur des possibilités de financements et de licence (valeur monnayable) et se révèlent toujours le résultat d'une collaboration intense avec le spécialiste (outil de collaboration).

En effet, le porteur du projet, lui-même issu de ce milieu, aura tendance à maintenir des contacts privilégiés avec les centres de recherche, par exemple en devenant leur partenaire industriel pour de nouveaux projets de recherche complémentaires pour l'activité de l'entreprise. Il reconstitue ainsi périodiquement cet avantage plutôt que de chercher à élargir ses débouchés. On comprend dès lors l'importance des brevets pour ce type d'entreprise de même que le peu d'intérêt pour les outils (marques, dessins et modèles) qui portent plus sur la relation avec les marchés.

\subsection{Innovation clé en mains}

Les PME «Innovation clé en mains » sont tout à fait particulières, car elles sont spécialisées dans la conception et l'innovation. Elles mettent au point de nouveaux produits, de nouveaux services, de nouvelles techniques, prennent des brevets, constituent de nouvelles sociétés pour effectuer les premiers développements et cherchent ensuite une entreprise prête à racheter le tout, afin d'en assurer l'exploitation commerciale. Elles agissent aussi directement sur mandat d'entreprises qui cherchent des solutions originales à leurs problèmes.

L'avantage compétitif de ces entreprises est leur inventivité et leur créativité. De taille plutôt petite, constituées avant tout d'ingénieurs et de designers, elles déploient leurs activités sur un large éventail de domaines, ce qui explique l'hétérogénéité des connaissances et des compétences de leur équipe de collaborateurs. La stratégie d'innovation est donc basée sur l'exploitation «pure» et aisée - en raison de la structure de la PME - de la créativité de l'équipe, couplée à la maîtrise de différentes technologies. Ce capital intellectuel est relayé par des contacts fréquents avec les hautes écoles techniques et les instituts universitaires.

L'utilisation des outils de PI est absolument centrale dans le fonctionnement de ces firmes. Elles en maîtrisent d'ailleurs tous les ressorts, grâce à leurs compétences internes (curiosité et autorégulation), mais aussi à des spécialistes externes (outil de collaboration), mûrement choisis et auxquels elles restent généralement fidèles. Le dépôt de brevets se fait systématiquement et ces derniers sont vendus aux clients (valeur immatérielle monnayable). C'est d'ailleurs ce qui fait la différence essentielle entre ces entreprises et le type «haute technologie» : ici, les inventeurs n'hésitent pas à se séparer du fruit de leurs recherches. Les créations sont donc déliées des personnes qui les ont portées, sont vendues ou cédées sous licence (avec une clause permettant généralement à la PME de continuer ses

Revue internationale P.M.E., vol. 18, n 2, 2005

(C) 2005 - Presses de l'Université du Québec

Édifice Le Delta I, 2875, boul. Laurier, bureau 450, Sainte-Foy, Québec G1V 2M2 - Tél.: (418) 657-4399 - www.puq.ca

Tiré de: Revue internationale P.M.E., vol. 18, n², sous la direction de Louis Raymond • PME1802N

Tous droits de reproduction, de traduction et d'adaptation réservés 
recherches sur le sujet) et deviennent des biens marchands. À cela s'ajoute naturellement une logique de communication, utilisant les titres de PI à la fois comme des vecteurs (publicité) et des sources d'informations (veille technologique).

\subsection{Automation et sous-traitance}

Les entreprises «Automation et sous-traitance» sont de taille plus importante, actives dans des industries traditionnelles comme la construction de machines ou le travail des métaux. Ce sont les clients - généralement des entreprises industrielles - qui formulent des demandes à haut contenu d'ingénierie. Des équipes organisées de R-D vont développer des solutions techniques qui feront l'objet de prises de brevets dans la mesure où elles sont originales. L'intérêt de cette prise de brevet est bien entendu d'empêcher l'imitation, mais aussi de se garantir la fidélité du client dans le futur. En effet, les séries de machines produites sont très courtes - il s'agit souvent de systèmes uniques - et la tentation de confier la réalisation de systèmes supplémentaires à des entreprises qui n'ont pas eu à supporter les frais de développement est là. Les entreprises de ce groupe exploitent donc des techniques traditionnelles dont elles assurent l'évolution constante au regard des tendances du marché et des besoins des clients.

L'organisation plus traditionnelle que dans les deux précédents groupes se retrouve dans la gestion de la PI. Les titres de PI servent à protéger les savoirs développés à l'interne: cela sera fait de manière efficace (une technique à maîtriser), en raison notamment des moyens internes à disposition (personnel spécialisé, budget pour ce faire, très peu de place à l'autorégulation) et maîtrisée (les licences, par exemple, sont généralement de type exclusif et ne laissent que peu de marge de manœuvre au preneur de licence). La logique de communication s'explique par l'effort important qui est mis dans la veille technologique et des marchés.

\subsection{Lièvres}

Les «lièvres» sont des entreprises de taille moyenne actives dans des branches traditionnelles. Au regard de différents aspects (organisation interne, taille des marchés, etc.), elles sont très semblables aux entreprises présentées dans le groupe précédent.

La seule et pertinente différence tient au fait que, par choix, elles n'ont pas de politique de PI. Cependant, elles utilisent la PI des autres, pratiquant une veille technologique intensive (outil de communication)! À partir de ces savoirfaire, les bureaux de R-D de ces entreprises s'attachent à défricher des terrains inexploités par les concurrents. Ils pensent et élaborent ainsi des produits, rapidement et de manière incrémentale. Ces entreprises considèrent que leur avantage

Revue internationale P.M.E., vol. 18, no 2, 2005

(C) 2005 - Presses de l'Université du Québec

Édifice Le Delta I, 2875, boul. Laurier, bureau 450, Sainte-Foy, Québec G1V 2M2 - Tél.: (418) 657-4399 - www.puq.ca

Tiré de: Revue internationale P.M.E., vol. 18, n² 2, sous la direction de Louis Raymond • PME1802N

Tous droits de reproduction, de traduction et d'adaptation réservés 
concurrentiel réside dans leur capacité à développer de nouvelles choses. Peu leur importe dès lors que d'autres entreprises s'inspirent par la suite de leurs produits: à ce moment-là, elles seront déjà en train de développer de nouveaux produits. Il devient, par conséquent, inutile de se protéger sur des marchés que l'on quitte rapidement.

«Nous préférons être ceux qui font la poussière que ceux qui la mangent derrière...»

Quelquefois, les démarches nécessaires pour une protection par la PI sont perçues comme longues, coûteuses et souvent vaines, bien que les entrepreneurs qui y renoncent en reconnaissent l'utilité dans certaines circonstances (contrats de licences à la clé, notamment). Ces PME, confiantes dans leurs relations durables et franches avec leurs clients et dans leur dynamique de création et d'innovation, préfèrent «toujours foncer» ou œuvrer «où les terrains sont en friche». Les brevets sont alors reconnus comme des sources d'informations fiables concernant les activités de la concurrence et utilisés comme tels, mais pas pour protéger la technologie propre de l'entreprise.

\subsection{Horlogerie et Cie}

Le groupe «Horlogerie et $\mathrm{C}^{\mathrm{ie}}$ » est composé d'entreprises de taille moyenne qui conçoivent et commercialisent des produits de consommation ayant un fort contenu esthétique, culturel et où la notoriété joue un rôle important. Elles s'adaptent périodiquement aux tendances et goûts des consommateurs tout en jouant sur des éléments constitués dans le passé (tradition, image de marque, symbolique, etc.). Une partie très importante de la valeur pour le consommateur réside non pas dans les opérations de transformation de la matière, mais bien dans le contenu immatériel du produit.

Les marques et les designs sont les titres les plus utilisés par ce type d'entreprise. Ces titres permettent des investissements sur l'image et de conserver le monopole sur cette symbolique essentielle (une technique à maîtriser). Les moyens mis à disposition sont importants et les démarches nécessaires sont le plus fréquemment effectuées par des spécialistes à l'interne. Comme on l'a vu plus haut, le «paraître» est fondamental: il s'agit d'exister aux yeux du public. La marque (marque, logo, etc.) permet de construire une notoriété et surtout une image propre tandis que les designs permettent de protéger les aspects esthétiques du produit. De plus, grâce à ces outils, il est possible de rendre cohérents ces différents éléments, cohérence qui constitue l'essentiel du capital de nombreuses entreprises horlogères (logique de communication). À noter que les brevets jouent ponctuellement un rôle important, que ce soit pour des fonctions techniques internes à la montre ou pour certains composants de l'habillage.

Revue internationale P.M.E., vol. 18, nº 2, 2005

(C) 2005 - Presses de l'Université du Québec

Édifice Le Delta I, 2875, boul. Laurier, bureau 450, Sainte-Foy, Québec G1V 2M2 • Tél.: (418) 657-4399 - www.puq.ca

Tiré de: Revue internationale P.M.E., vol. 18, $\mathrm{n}^{\circ} 2$, sous la direction de Louis Raymond - PME1802N

Tous droits de reproduction, de traduction et d'adaptation réservés 


\subsection{Biens de consommation}

Dans le groupe «Biens de consommation», nous avons réuni les PME dont les produits ou services s'adressent directement aux consommateurs et qui, à la différence de l'ensemble précédent, n'ont pas un contenu immatériel très important.

Ces PME ont été amenées à recourir à la propriété intellectuelle dans des circonstances précises et ponctuelles. Elles s'inscrivent dans un cadre économique traditionnel; ce peut être des fabricants de friteuses, des «boucheries», voire des menuiseries... Elles ne tirent aucun avantage concurrentiel particulier du fait de l'utilisation des outils de PI. Cependant, une invention ou un nouveau logo est un «fait d'armes» qu'il s'agit de communiquer (article dans la presse, marchandisage, sponsoring, etc.) et d'exposer dans la salle de réception! Marques et brevets sont donc des «filets» à leur politique de marketing.

«Regardez, le brevet est exposé au mur, derrière vous!»

... nous fait remarquer, au début de l'entretien, un chef d'entreprise.

La PME visitée conçoit et produit des ustensiles de cuisine. Ses clients sont dispersés à travers le monde et vont du voisin de palier aux chaînes multinationales de restauration. Le brevet n'a pas révolutionné la production de l'entreprise. Il est perçu comme une «plus-value» par rapport aux activités industrielles et commerciales habituelles. Dès lors, il s'agit de le «vendre» comme tel. Pour cette PME, le brevet est non seulement une manière de «formaliser l'avantage technologique pris sur les concurrents », mais aussi de rendre compte, pour les clients ou le grand public, de son dynamisme.

\section{Conclusion}

Sur le plan juridique, la propriété intellectuelle offre nombre d'avantages par rapport à des règlements tacites ou contractuels. Cependant, la mise en œuvre effective de ces différents droits par les PME se heurte à deux problèmes.

Premièrement, les raisons du recours à ces outils ne sont pas immédiates, car la protection, qui paraît être l'objectif évident au premier abord, se révèle dans les faits toujours incomplète et contournable. De plus, elle est très difficile à mettre en œuvre face à des acteurs économiques plus puissants. Dès lors, pourquoi les PME utilisent-elles ces outils et recourent néanmoins à la PI? Les pratiques des PME se caractérisent par des logiques différentes, répondant chacune à des préoccupations essentielles de leurs dirigeants. Ces logiques s'apparentent à différentes «pièces d'un puzzle» qu'il s'agit de bien ordonner entre elles pour en faire un ensemble cohérent et efficace. La considération d'une seule de ces

Revue internationale P.M.E., vol. 18, nº 2, 2005

(c) 2005 - Presses de l'Université du Québec

Édifice Le Delta I, 2875, boul. Laurier, bureau 450, Sainte-Foy, Québec G1V 2M2 - Tél.: (418) 657-4399 - www.puq.ca

Tiré de: Revue internationale P.M.E., vol. 18, n 2, sous la direction de Louis Raymond • PME1802N

Tous droits de reproduction, de traduction et d'adaptation réservés 


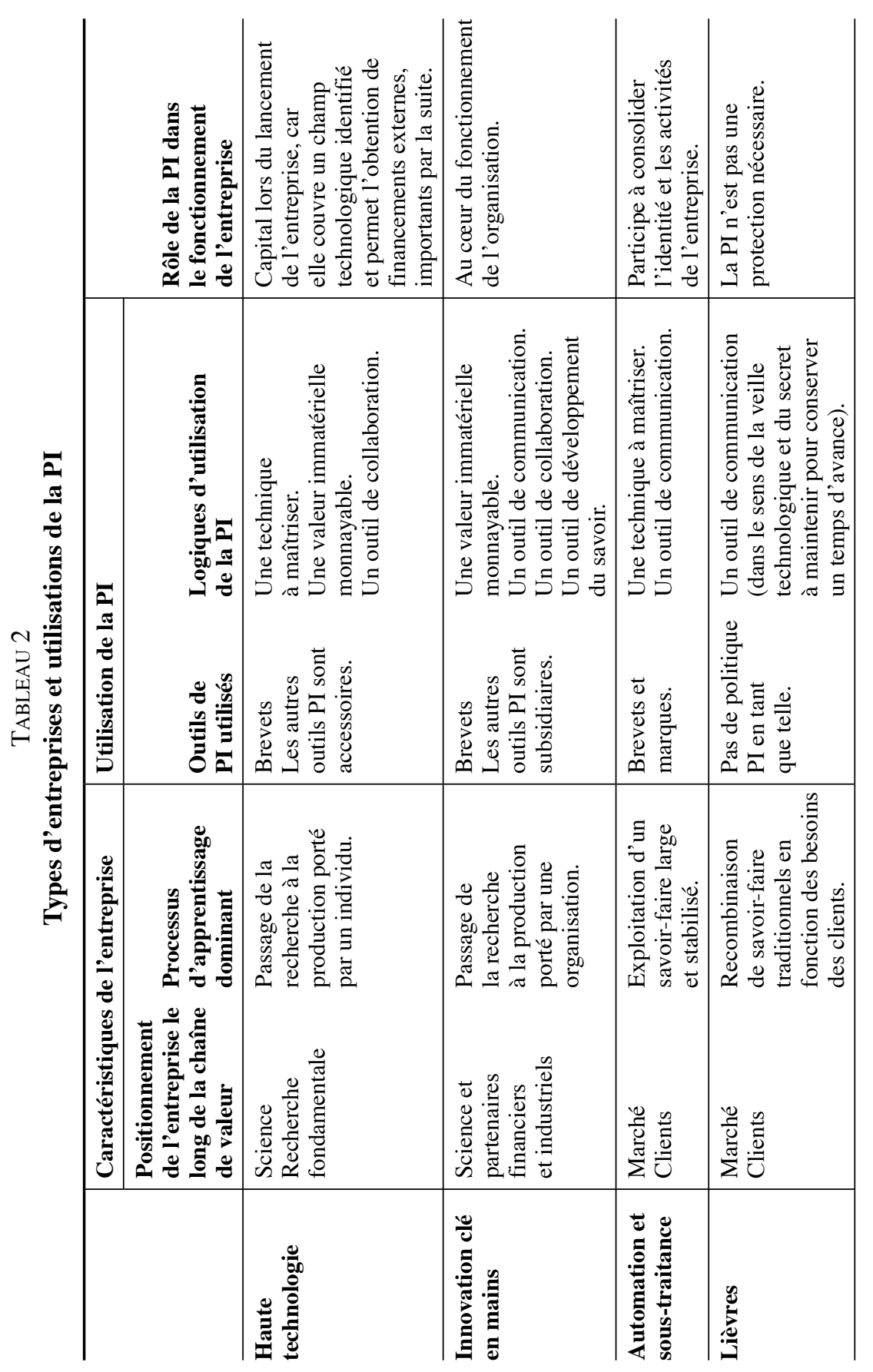

Revue internationale P.M.E., vol. 18, $\mathrm{n}^{\mathrm{o}}$ 2, 2005

(c) 2005 - Presses de l'Université du Québec

Édifice Le Delta I, 2875, boul. Laurier, bureau 450, Sainte-Foy, Québec G1V 2M2 • Tél.: (418) 657-4399 - www.puq.ca

Tiré de: Revue internationale P.M.E., vol. 18, n² 2, sous la direction de Louis Raymond • PME1802N

Tous droits de reproduction, de traduction et d'adaptation réservés 


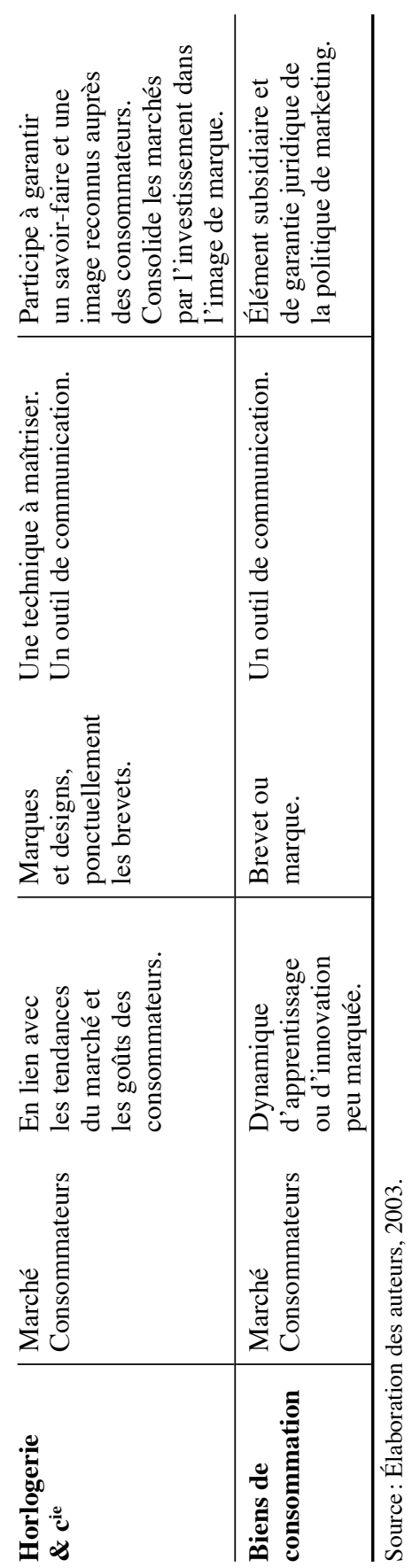

Revue internationale P.M.E., vol. 18, nº 2, 2005 
fonctions, aussi approfondie soit-elle, ne semble guère satisfaisante. Par exemple, la PME qui n'utilise la PI que dans sa seule fonction de protection s'expose à des désillusions. Cela est d'autant plus important pour de petites entités industrielles ou de recherche, qu'elles n'ont, généralement, peu ou pas de ressources pour faire respecter leurs droits, contrairement à de plus grandes entreprises. Finalement, ces logiques rendent compte des différences existant entre les PME et entre les divers stades de leur développement. Ainsi, le brevet est un moyen utilisé lors du lancement de nouveaux projets industriels alors que la marque permet de stabiliser le marché de produits existants.

Deuxièmement, il n'existe pas de solution simple, standard, adaptée à toute PME. La mise en œuvre de ces outils demande donc une certaine maîtrise par l'entreprise qui choisira la logique d'utilisation correspondant à ses besoins, quitte à recourir ensuite à d'autres logiques au gré des besoins du développement de ses produits et de ses compétences. Dès lors, quelles sont les modalités de cet apprentissage et quelle est sa place dans le développement plus général de l'entreprise? Comme dans d'autres domaines, les PME sont largement tributaires du réseau de compétences dont elles s'entourent. Une politique de PI ne s'élabore pas du jour au lendemain et elle mobilise des compétences diverses (ingénieurs-conseils, avocats, services de veille technologique, organismes de transfert de technologie, etc.). Comme tout service à haute valeur ajoutée vendu aux entreprises, l'expertise en PI suppose, de la part de l'expert, une bonne connaissance de l'activité spécifique de l'entreprise et de son environnement, ainsi qu'une connaissance et une pratique de base de la PI de la part de la PME. Par conséquent, les essais et erreurs semblent difficilement évitables. C'est certainement pour cette raison que les PME rencontrées semblent privilégier des relations sur le long terme: si la PME est amenée à appréhender toujours plus ces outils, il existe un apprentissage dans la maîtrise de ces droits et de leurs modalités.

Même si brevets, marques, designs et droits d'auteur, dans une moindre mesure, ne sont pas des solutions «miracles», même s'ils ne changent pas les rapports de force entre les PME et les grandes entreprises, ils sont indubitablement des moyens qui permettent d'affirmer ou de consolider l'autonomie des petites entreprises qui les maitrisent. Le recours aux droits de PI permet d'identifier concrètement les inventions et les créations, mais aussi les besoins et les ressources des différents acteurs. À partir de là, les portefeuilles de PI deviennent des instruments de gestion de projet et de gestion stratégique. Ils renforcent l'emprise de l'entreprise sur un domaine technologique, une image, un produit ou un service. En plus de rendre certains investissements économiquement possibles et plus sûrs, ils permettent de réduire ou de «baliser» les incertitudes liées à l'innovation et à la création. À ce jour, ils sont d'autant plus déterminants que 
la production devient de plus en plus immatérielle et que l'innovation joue un rôle important. Ils sont de plus en plus les supports d'une économie de marché moderne, l'économie du savoir.

D'un point de vue pratique, l'approche proposée ici permet à une PME d'utiliser ces différents droits comme un « arc à plusieurs cordes ». Cette démarche n'est pas exhaustive, mais elle a le mérite de montrer que les PME peuvent recourir à la PI avec succès et les encourage dans ce sens. Les portefeuilles de PI et certains de leurs mécanismes joints (contrats de licence, veille technologique, etc.) sont des sources de connaissances, des canaux de transferts de savoirs, l'expression de formes de maturité et de stabilité dans les relations entre acteurs. Ces aspects ne doivent pas être négligés par les PME.

Enfin, quelles pistes de recherche peut-on évoquer à partir de ce travail? Nous n'avons considéré que des logiques «pures». La démarche des économies de la grandeur va plus loin dans le sens où elle débouche sur la combinaison des différentes logiques d'où émergent certaines configurations, appelées «compromis». L'entreprise parvient-elle à concilier ces différentes logiques? Débouchet-on sur des combinaisons plus ou moins stables, caractéristiques d'un type d'entreprise ? Les rapports de force entre les PME et des entreprises plus puissantes sont une autre piste qui n'a été qu'évoquée ici, mais qui joue, de toute évidence, un rôle déterminant. Pour cela, il faudrait mobiliser d'autres outils théoriques que ceux des économies de la grandeur, car ces dernières partent de l'idée que les logiques à l'œuvre dans la société peuvent se comprendre du moment qu'elles sont justifiables, éthiquement fondées... On voit bien là la limite de l'approche!

\section{Annexe I}

Les différents droits de propriété intellectuelle énumérés au point 1.1. méritent un bref éclairage. Le tableau présenté à la fin de cette annexe résume les principales propriétés de chacun de ces droits.

\section{Le brevet}

Le brevet permet de protéger les inventions. Ce sont des créations réalisées par l'homme et qui apportent quelque chose de nouveau à l'état de la technique. $A$ contrario, les lois de la nature, les phénomènes physiques et les idées abstraites ne peuvent pas faire l'objet d'un brevet en tant que tels. Le brevet protège donc la matérialisation-concrétisation d'idées ou de lois de la nature dans une application dotée d'un effet technique et concret. Le brevet confère à son titulaire un droit exclusif d'utiliser son invention et la possibilité d'empêcher les tiers d'en faire usage professionnellement. Les limites à ce monopole s'expriment de deux

Revue internationale P.M.E., vol. 18, n 2, 2005

(C) 2005 - Presses de l'Université du Québec

Édifice Le Delta I, 2875, boul. Laurier, bureau 450, Sainte-Foy, Québec G1V 2M2 • Tél.: (418) 657-4399 - www.puq.ca

Tiré de: Revue internationale P.M.E., vol. 18, n² 2, sous la direction de Louis Raymond • PME1802N

Tous droits de reproduction, de traduction et d'adaptation réservés 
manières : le champ d'application du brevet est limité (durée, territoire et domaine d'activité) et le titulaire est tenu de publier le contenu de son brevet qui s'accompagne ainsi d'une diffusion de l'information technique concernée. La loi prévoit aussi des licences obligatoires pour lutter contre d'éventuelles «utilisations perverses » du brevet visant à créer des pénuries artificielles sur le marché.

\section{La marque}

La marque confère une identité à des services ou des produits. Elle permet de différencier les prestations d'une entreprise d'autres services ou produits du même genre se trouvant sur le marché et offerts par d'autres entreprises. Elle est ainsi un signe distinctif et qui, après son enregistrement, est protégé. La marque attribue à son titulaire un droit exclusif d'utiliser ce qui a fait l'objet de la protection: il s'agit généralement d'un nom, composé indifféremment de chiffres ou de lettres, d'un logo (statique ou animé), plus rarement de formes ou de sons - jingles, par exemple - ou, dans des cas très rares et particuliers, de couleurs ou d'odeurs.

\section{Les designs}

Les designs, selon la terminologie de la nouvelle loi suisse, englobent les dessins et modèles industriels. Ils protègent les créations caractérisées à la fois par un aspect esthétique et utilitaire. Les dispositions de cette loi sont hybrides, reprenant tantôt, par certains raisonnements ou modalités, des caractéristiques rappelant celles du droit d'auteur (aspect lié à l'originalité, bien que l'individualité du droit d'auteur soit plus stricte que celui de l'originalité des designs) et tantôt des caractéristiques se rapprochant du droit des brevets (exigence de nouveauté, même si elle est comprise de manière moins stricte en droit des designs qu'en matière de brevet). La loi sur les designs s'applique à toute création graphique (2D - tapisseries, emballages décoratifs, cadrans de montres, étiquettes, tissus...) ou plastique (3D - lampes, montres, bouteilles, machines à café, services de tables, pièces montées, etc.). De manière générale, nombre d'objets familiers nous entourant peuvent être protégés par le droit des designs, dès qu'ils sont le reflet d'une certaine recherche esthétique.

\section{Le droit d'auteur}

Le droit d'auteur protège les œuvres qui ont un caractère individuel et qui sont l'expression de la pensée humaine. La loi énumère, sans prétention d'exhaustivité, les différentes catégories d'œuvres susceptibles d'être protégées. Ce sont, en particulier, les œuvres littéraires (livres, brochures, discours, etc.), les œuvres artistiques (peintures, sculptures, lithographies, etc.), les œuvres musicales, les œuvres d'architecture et des arts appliqués, les œuvres photographiques, cinématogra- 
phiques ou audiovisuelles, les œuvres chorégraphiques, les œuvres techniques (cartes géographiques, topographiques, etc.) ou scientifiques, les programmes d'ordinateurs (logiciels). Les droits d'auteur naissent ipso facto de la création même de l'œuvre et protègent à la fois la dimension personnelle de l'auteur (à travers les droits moraux) et l'aspect patrimonial de l'exploitation de l'œuvre (à travers les droits patrimoniaux).

\section{Les indications géographiques}

Les indications géographiques sont des noms de lieu ou la référence à un lieu, identifiant des produits, originaires de ce milieu géographique précis et possédant des qualités ou une réputation particulière dues à cette origine. Ce sont donc des droits de propriété intellectuelle, somme toute assez proches dans leur conception des marques de garantie. Cependant, elles ne s'appliquent pas obligatoirement à l'ensemble des produits, puisqu'il doit être reconnu que l'origine du bien est déterminante pour sa qualité et ses caractéristiques. Ces indications sont d'une importance primordiale pour les biens agricoles ou de consommation; mais peu de produits industriels peuvent à ce jour revendiquer une telle protection. À noter que les indications de provenance géographique ou les appellations d'origine, utilisés pour des produits agricoles ou élaborés à partir de matières premières issues de l'agriculture sont régies par la loi sur l'agriculture (art. 16). Sur cette base, elles sont soumises à une procédure d'enregistrement et à l'élaboration d'un cahier des charges strict, spécifiant les qualités ou caractéristiques particulières des produits considérés; un processus de contrôle est aussi mis en place par la loi. Pour leur part, les indications de provenance industrielles sont réglées par la loi sur les marques et ne sont pas soumises à une procédure d'enregistrement.

Revue internationale P.M.E., vol. 18, nº 2, 2005

(c) 2005 - Presses de l'Université du Québec

Édifice Le Delta I, 2875, boul. Laurier, bureau 450, Sainte-Foy, Québec G1V 2M2 • Tél.: (418) 657-4399 - www.puq.ca

Tiré de: Revue internationale P.M.E., vol. 18, n² 2 , sous la direction de Louis Raymond P PME1802N

Tous droits de reproduction, de traduction et d'adaptation réservés 


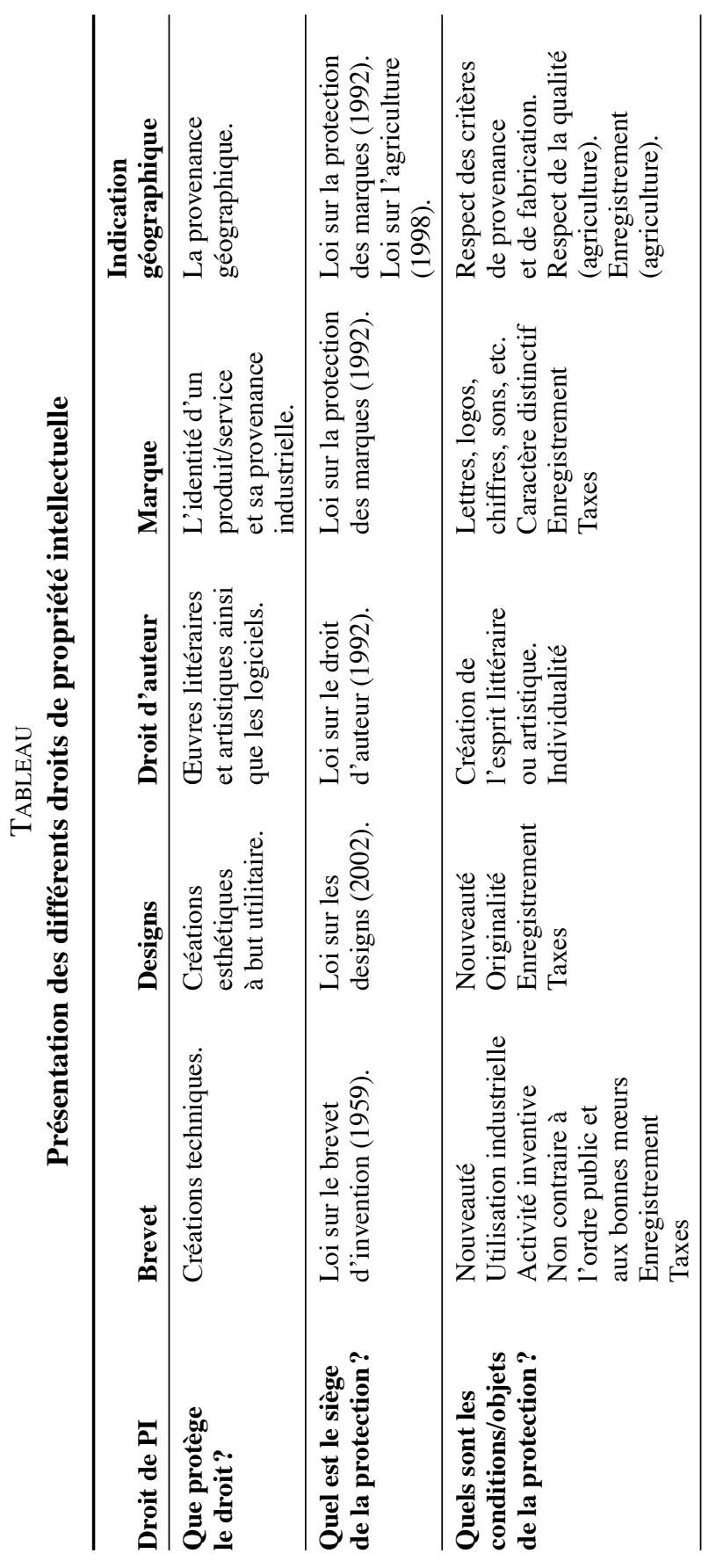

Revue internationale P.M.E., vol. 18, $\mathrm{n}^{\circ}$ 2, 2005

(C) 2005 - Presses de l'Université du Québec

Édifice Le Delta I, 2875, boul. Laurier, bureau 450, Sainte-Foy, Québec G1V 2M2 • Tél.: (418) 657-4399 - www.puq.ca

Tiré de: Revue internationale P.M.E., vol. 18, n² 2, sous la direction de Louis Raymond • PME1802N

Tous droits de reproduction, de traduction et d'adaptation réservés 


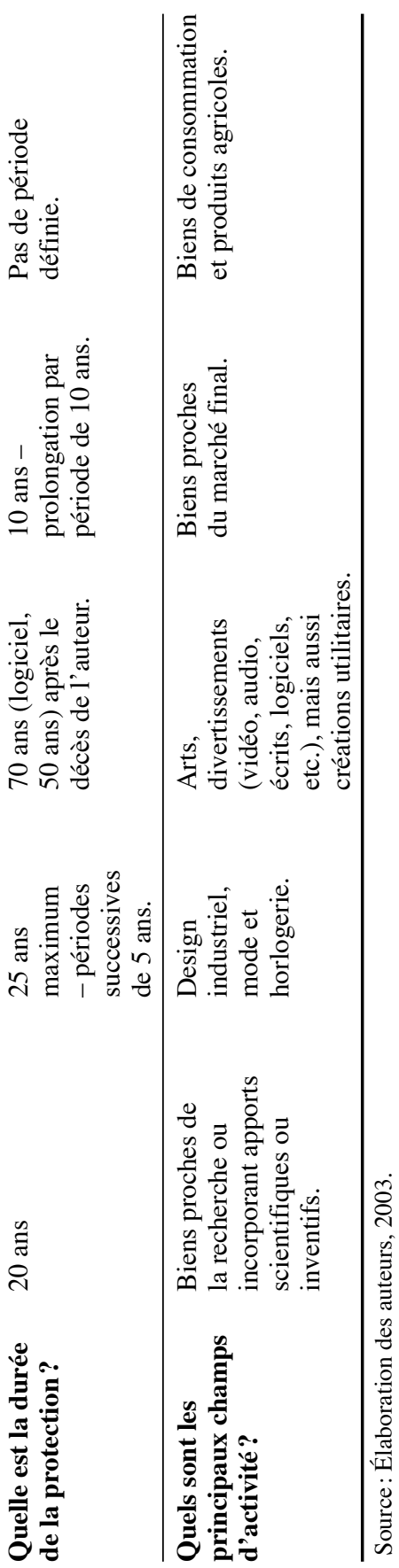

Revue internationale P.M.E., vol. 18, nº 2, 2005 


\section{Bibliographie}

AMABle, B., R. BARré et R. BOyer (1997), Les systèmes de l'innovation à l'ère de la globalisation, Paris, Economica.

AllegrezZa, S. et A. Guarda-Rauchs (1999), «The determinants of trademark deposits: an econometric investigation », Économie appliquée, tome LII, no 2, p. 51-68.

Baumol, W.J., J. Panzar et R. Willig (1982), Contestable Markets and the Theory of Industry Structure, New York, Harcourt Brace Jovanovich.

BESSY, C. et E. BRousseAu (1997), «Brevet, protection et diffusion des connaissances : une relecture néo-institutionnelle des propriétés de la règle de droit», Revue d'économie industrielle, $\mathrm{n}^{\circ}$ 79, p. 244-260.

Boltanski, L. et L. ThÉvenot (1991), De la justification, les économies de la grandeur, Paris, Gallimard.

BRENNER, C. (1998), «La propriété intellectuelle et ses conséquences pour les pays en voie de développement», Annuaire suisse - Tiers monde IUED, n 17, p. 7-24.

CHERPILlod, I. (1985), L'objet du droit d'auteur, Lausanne, CEDIDAC.

Crevolsier, O. (2001), «L'approche par les milieux innovateurs : état des lieux et perspectives », RERU, n ${ }^{\circ} 1$, p. 153-166.

DAVIS, L. (2001), «Can the patent system continue to adapt to the modern global knowledge economy», Exposé présenté lors du Colloque «Nelson et Winter», Ålborg, Danemark, 12-15 juin.

DefFains, B. (1997), «Progrès scientifique et analyse économique des droits de propriété intellectuelle», Revue d'économie industrielle, no 79, p. 95-118.

De Rosnay, J. (2000), L'homme symbiotique, Regards sur le troisième millénaire, Paris, Seuil.

FORAY, D. (1995), «Les brevets dans la nouvelle économie de l'innovation», Changement institutionnel et changement technologique - Évaluation, droits de propriété intellectuelle, système national d'innovation, CNRS (M. Baslé, D. Dufourt, J.-A. Héraud et J. Perrin), p. 119-149.

Granstrand, O. (1999), The Economics and Management of Intellectual Property, Londres, Edward Elgar.

HÉRAUD, J.-A. (1995), «Brevets et contexte institutionnel de la création technologique», Changement institutionnel et changement technologique - Évaluation, droits de propriété intellectuelle, système national d'innovation, Paris, CNRS (M. Baslé, D. Dufourt, J.-A. Héraud et J. Perrin), p. 91-117.

LE BAS, C. (1999), «Matériaux pour une économie de la propriété intellectuelle: problématique, perspectives et problèmes », Économie appliquée, tome LII, no 2, p. 19-50.

Livian, Y.-F. et G. Herreros (1994), «L'analyse des économies de la grandeur: une nouvelle grille d'analyse des organisations?», Revue française de gestion, 101, p. $43-59$.

Merges, R.P., P.S. Menell et M.A. Lemey (2000), Intellectual Property in the New Technological Age, $2^{\mathrm{e}}$ édition, New York, Aspen Law \& Business.

Revue internationale P.M.E., vol. 18, nº 2, 2005

(C) 2005 - Presses de l'Université du Québec

Édifice Le Delta I, 2875, boul. Laurier, bureau 450, Sainte-Foy, Québec G1V 2M2 • Tél.: (418) 657-4399 - www.puq.ca

Tiré de: Revue internationale P.M.E., vol. 18, $\mathrm{n}^{\circ} 2$, sous la direction de Louis Raymond - PME1802N

Tous droits de reproduction, de traduction et d'adaptation réservés 
Primo Barga, C.A., C. Fink et C. Paz Sepuldeva (2000), Intellectual Property Rights and Economic Development, Washington, DC, World Bank Discussion Paper no 412.

RIFKIN, J. (2000), L'âge de l'accès, Paris, La Découverte.

STEINMÜLlER, W.E. (1999), Networked Knowledge and Knowledge Based Economies, Delft, Telematica Institut.

WORKING PAPER (2001), «Intellectual property rights and small and medium-sized entreprises: an agenda for the future », Document de travail d'un groupe d'experts et officiels en vue de la conférence de l'OMPI sur le thème PI-PME, Milan, 9-10 février.

Revue internationale P.M.E., vol. 18, $\mathrm{n}^{\circ}$ 2, 2005

(C) 2005 - Presses de l'Université du Québec

Édifice Le Delta I, 2875, boul. Laurier, bureau 450, Sainte-Foy, Québec G1V 2M2 • Tél.: (418) 657-4399 - www.puq.ca

Tiré de: Revue internationale P.M.E., vol. 18, no 2, sous la direction de Louis Raymond • PME1802N

Tous droits de reproduction, de traduction et d'adaptation réservés 
(C) 2005 - Presses de l'Université du Québec

Édifice Le Delta I, 2875, boul. Laurier, bureau 450, Sainte-Foy, Québec G1V 2M2 • Tél.: (418) 657-4399 - www.puq.ca

Tiré de: Revue internationale P.M.E., vol. 18, $\mathrm{n}^{\circ}$ 2, sous la direction de Louis Raymond - PME1802N

Tous droits de reproduction, de traduction et d'adaptation réservés 